\title{
Rubinstein-Taybi Syndrome: spectrum of CREBBP mutations in Italian patients
}

Angela Bentivegna ${ }^{1}$, Donatella Milani ${ }^{2}$, Cristina Gervasini ${ }^{1}$, Paola Castronovo ${ }^{1}$, Federica Mottadelli ${ }^{1}$, Stefano Manzini ${ }^{3}$, Patrizia Colapietro ${ }^{3}$, Lucio Giordano ${ }^{4}$, Francesca Atzeri ${ }^{2}$, Maria T Divizia ${ }^{5}$, Maria L Giovannucci Uzielli ${ }^{6}$, Giovanni Neri ${ }^{7}$, Maria F Bedeschi ${ }^{8}$, Francesca Faravelli ${ }^{9}$, Angelo Selicorni ${ }^{\dagger 2}$ and Lidia Larizza* ${ }^{* 1}$

Address: ${ }^{1}$ Division of Medical Genetics, San Paolo School of Medicine, University of Milan, Italy, ${ }^{2}$ I Clinica Pediatrica, Fondazione Ospedale Maggiore Policlinico Mangiagalli e Regina Elena, Milan, Italy, ${ }^{3}$ Department of Biology and Genetics, Medical Faculty, University of Milan, Milan, Italy, ${ }^{4}$ Neuropsichiatria Infantile, Spedali Civili, Brescia, Italy, ${ }^{5}$ Laboratory of Molecular Genetics, G. Gaslini Institute, Genova, Italy, ${ }^{6}$ Department of Paediatrics, Genetics Unit - Children's Hospital A. Meyer, Florence, Italy, 7 Institute of Medical Genetics, Catholic University, Rome, Italy, ${ }^{8}$ Servizio di Genetica Medica, Fondazione Ospedale Maggiore Policlinico Mangiagalli e Regina Elena, Milan, Italy and ${ }^{9}$ Department of Human Genetics of Galliera Hospital, Genoa, Italy

Email: Angela Bentivegna - angela.bentivegna@unimib.it; Donatella Milani - donatella.milani@icp.mi.it; Cristina Gervasini - cristina.gervasini@unimi.it; Paola Castronovo - paola.castronovo@gmail.com; Federica Mottadelli - federica_mottadelli@hotmail.com; Stefano Manzini - heartofwinter@email.it; Patrizia Colapietro - patrizia.colapietro@unimi.it; Lucio Giordano - pavifan@tin.it; Francesca Atzeri - francesca.atzeri@tiscali.it; Maria T Divizia - maridiv@tin.it; Maria L Giovannucci Uzielli - uzielli_ml@unifi.it; Giovanni Neri - gneri@rm.unicatt.it; Maria F Bedeschi - m.f.bedeschi@icp.mi.it; Francesca Faravelli - francesca.faravelli@galliera.it; Angelo Selicorni - ambulatorio@gencli.it; Lidia Larizza* - lidia.larizza@unimi.it

* Corresponding author †Equal contributors

Published: 19 October 2006

BMC Medical Genetics 2006, 7:77 doi:10.1 186/147/-2350-7-77
Received: 02 August 2006

Accepted: 19 October 2006

This article is available from: http://www.biomedcentral.com/I47/-2350/7/77

(c) 2006 Bentivegna et al; licensee BioMed Central Ltd.

This is an Open Access article distributed under the terms of the Creative Commons Attribution License (http://creativecommons.org/licenses/by/2.0), which permits unrestricted use, distribution, and reproduction in any medium, provided the original work is properly cited.

\begin{abstract}
Background: Rubinstein-Taybi Syndrome (RSTS, MIM 180849) is a rare congenital disorder characterized by mental and growth retardation, broad and duplicated distal phalanges of thumbs and halluces, facial dysmorphisms and increased risk of tumors. RSTS is caused by chromosomal rearrangements and point mutations in one copy of the CREB-binding protein gene (CREBBP or CBP) in 16p I3.3. To date mutations in CREBBP have been reported in $56.6 \%$ of RSTS patients and an average figure of $10 \%$ has ascribed to deletions.

Methods: Our study is based on the mutation analysis of CREBBP in 3 I Italian RSTS patients using segregation analysis of intragenic microsatellites, BAC FISH and direct sequencing of PCR and RTPCR fragments.

Results: We identified a total of five deletions, two of the entire gene and three, all in a mosaic condition, involving either the $5^{\prime}$ or the $3^{\prime}$ region. By direct sequencing a total of 14 de novo mutations were identified: 10 truncating ( 5 frameshift and 5 nonsense), one splice site, and three novel missense mutations. Two of the latter affect the HAT domain, while one maps within the conserved nuclear receptor binding of (aa I-170) and will probably destroy a Nuclear Localization Signal. Identification of the p.Asn 1978Ser in the healthy mother of a patient also carrying a de novo frameshift mutation, questions the pathogenetic significance of the missense change reported as
\end{abstract}


recurrent mutation. Thirteen additional polymorphisms, three as of yet unreported, were also detected.

Conclusion: A high detection rate (6I.3\%) of mutations is confirmed by this Italian study which also attests one of the highest microdeletion rate (16\%) documented so far.

\section{Background}

Rubinstein-Taybi syndrome (RSTS; MIM 180849) is a congenital disorder characterized by growth retardation and psychomotor developmental delay, broad and duplicated distal phalanges of thumbs and halluces, and a wide range of typical dysmorphic features. Facial dysmorphology includes down-slanted palpebral fissures, broad nasal bridge, beaked nose and micrognathia [1]. In addition, patients with RSTS have an increased, although not well documented, risk of tumor formation $[2,3]$. RSTS was shown to be associated with microdeletions and point mutations in the gene encoding the CREB-binding protein (CREBBP, also known as "CBP"), located in 16p13.3 [4]. CREBBP is a transcriptional coactivator and possesses acetyltransferase activity on lysine residues of histones and nonhistone proteins [5]. CREBBP partecipates in basic cellular functions, including growth, differentiation, DNA repair and apoptosis, regulating the expression of many genes [6]. The mutations found in RSTS patients vary from point mutations to relatively large microdeletions, which remove the entire gene, attesting that haploinsufficiency of this dosage-sensitive gene is the ultimate cause of the syndrome. More precisely, studies of patients with missense and splice-site mutations that affect only the histone acetyl transferase (HAT) domain of CREBBP demonstrated that loss of HAT activity is sufficient to cause the syndrome $[7,8]$. The related p300 protein, which is encoded by the EP300 gene on chromosome 22q13.2, shares homology with CREBBP and accordingly serves as a transcriptional coactivator endowed with a HAT domain [9]. Mutations of EP300 have been recently reported in a small subset $(3.3 \%)$ of RSTS patients [10]. This figure and the low detection rates (from $40 \%$ to $56.6 \%$ ) of CREBBP gene assessed by all mutations studies of RSTS patients suggest that other candidate genes might contribute to the pathogenesis of this syndrome $[4,10$ 12]. We recruited 31 patients with consistent RSTS clinical diagnosis and we tested them by microsatellite segregation analysis, FISH, RT-PCR and direct sequencing to find mutations of the CREBBP gene. This multimethod approach allowed us to identify five deletions and 14 point mutations, accounting for the highest $(61.3 \%)$ mutation rate so far assessed. Particularly FISH analysis was instrumental for detecting even subtle microdeletions proving to be a method suitable more than mutation analysis to disclose low level mosaic conditions.

\section{Methods Subjects}

We analysed samples of 31 unrelated patients sent to our laboratory with parental consent with the request of genetic analysis for Rubinstein-Taybi syndrome. Studies and procedures were in accordance with the ethical standards of the host institutions. The group consisted of 16 female and 15 male patients, aged 2 to 42 years. Clinical diagnoses were established by different Centers and eventually confirmed by the coordinating clinical Center (Dept of Pediatrics, University of Milan) based on clinical records and photographs, when no pathogenetic mutation could be found. Careful examination of the patients' phenotypes was done, and the presence of malformations and medical complications were confirmed or excluded.

Thirty subjects had a normal karyotype by Giemsa-trypsin banding at 450-band resolution; patient 38 showed a 46 , XY, der(14)t(9;14)(p11.2;p11.2) karyotype.

\section{Cell cultures, chromosome and interphase nuclei preparations}

Phytohemagglutinin-stimulated peripheral blood lymphocytes were set up in culture from samples using Chromosome Kit "Synchro" (Celbio) and modified RPMI (Irvine Scientific) plus 5\% fetal calf serum (Gibco). The cultures were blocked with colchicine after $72 \mathrm{~h}$. Chromosome preparations were obtained using a standard technique.

\section{Clone preparations}

BAC clones RP11 95J11, RP11 461 A8 and RP11 566K11 were supplied by Mariano Rocchi [13]; BAC RP11 1072J2 was purchased from the CHORI BAC PAC Resource Center (Oakland, USA). DNA was obtained starting from a single colony grown in $4 \mathrm{ml}$ of LB medium supplemented with $20 \mu \mathrm{g} / \mathrm{ml}$ chloramphenicol (Sigma) following standard procedures.

\section{FISH}

BAC clones were labelled with digoxigenin-dUTP (Roche Diagnostic) using a nick translation kit (Roche Diagnostic). The FISH experiments were performed according to standard procedures [14]. The chromosomes were counterstained with DAPI in antifade (Vectashild), and then visualised using a Leitz DM-RB microscope equipped for DAPI and FITC/TRITC epifluorescence optics. The images were captured by means of a CCD camera (Hamamatsu 
3CCD Camera, C5810) and visualised using Highfish software (Casti Imaging).

\section{Analysis of FISH signals in mosaic condition}

Almost 50 metaphases and 100 nuclei were scored for each sample in order to establish the mosaic condition. Only intact and undamaged nuclei free of cytoplasm were analyzed. Nuclei with low signal intensities, diffuse signals, or absence of signals on both homolog chromosomes were considered to be hybridization failures and were not scored. Two small focal (or paired) signals of the same color and the same intensity, separated by a distance of less than the area of one signal, were considered to be a split signal from one chromosome. Interphase nuclei with one large signal of the same color and increased intensity of fluorescence with the absence of a second hybridization signal in an interphase nucleus were considered to be overlapping (or over-position) of two signals and were not scored. Informative mosaic samples were defined as those in which more than $5 \%$ of the nuclei had a reproducible abnormal pattern of signals different from normal chromosomal signals.

\section{Microsatellite segregation analysis}

Fluorescent genotyping was performed as previously described [11]. The following fluorescently dye-labeled chromosome 16p microsatellite markers: MS4, MS2 and D16S3065 (from centromere to telomere) were used for segregation analysis from parents to probands. Fluorescence detection was performed on an ABI 3100 sequencer. ABI PRISM software (Genescan) was used for gel analysis.

\section{RT-PCR analysis}

RT-PCR analysis was performed as previously described [11] for patients 14, 16, 20, 25, 28 and 29 for whom mRNA was available.

\section{Molecular analysis}

The DNA sequencing of all coding exons of the CREBBP gene was performed as previously described [11]. Twentysix CREBBP PCR genomic fragments were amplified (see Additional file 1). The DNA sequencing protocol was updated for use with the BigDye Chemistry, Versions 2.0 and 3.1, and the ABI PRISM 310 and 377 Genetic analyzer systems (Applied Biosystems, Darmstadt, Germany). If a mutation or polymorphism was found, a second sequencing run was performed for confirmation. The origin of the mutation or polymorphism, whether de novo or familial, was established if parental DNA was available. Paternity testing was not performed due to legal restrictions. The Human Gene Mutation Database (HGMD) [15], recent publications [8,10-12], and the dbSNP database [16] were consulted to check whether sequence variations were previously reported or not. Missense mutations of patients 15 and 46 identified in this study were absent in 100 con- trols, while that carried by patient 23 was absent in 50 controls.

\section{Results Clinical evaluation}

Thirty-one Italian RSTS patients, whose main signs are reported in Table 1, were carefully clinically analysed. Sex ratio was $\mathrm{F}: M=16: 15$. All patients had psychomotor/ mental retardation, which ranged from mild to severe in 17 cases. Microcephaly was evident in 20 (64.5\%); a subset ( 12 cases) accounting for $61.3 \%$ of RSTS patients manifested poor growth. The main facial features were specifically described and/or evident from photos (see Figure 1) and the overall facial gestalt was absolutely typical in all but one patient. As reported in Table 1, the typical large thumb was evident in 93\% (27/29 patients), and in 11 cases it was also abducted; the hallux was large in $89 \%(26 / 29)$, and only in 4 cases it was valgus/varus. Only 11 individuals had major malformations of internal organs or limbs: congenital heart disease (patent ductus arteriosus, ventricular/atrial septum defects, bicuspid aorta) was reported in 6 of them, cryptorchid testes in 3, coloboma of the optic nerve, renal hypoplasia, duodenal malrotation and hexadactyly in one. In 5 patients supernumerary nipples were evident. Only in two cases (pt 16 and 17) lacrimal ducts stenosis and sensorineural deafness (pt 14 and 38) were signaled. In case 46 the presence of multiple epitheliomas was reported. In another single case (pt 15) Perthe's disease was found.

\section{Mutation analysis}

We screened the 31 recruited patients for point mutations, small deletions or insertions, and large deletions and duplications of the CREBBP gene. First, by genotyping the polymorphic intragenic repeats MS4, MS2 and D16S3065 [11] plus one SNP (c.5454G>A, [12]) we could identify two deletions spanning the whole gene in patients 30 and 41. Both deletions affected the maternal chromosome. Figure 2 shows for patient 30: (A) the haplotype reconstruction attesting the lack of three maternally contributed intragenic polymorphic loci; (B) FISH of the CREBBP spanning BAC 1072J2 evidencing a signal of reduced intensity on one chromosome 16. By means of FISH analysis we detected three additional deletions, all in a mosaic condition. Two (pt 40, pt 66) affect the 5' of the gene, while one (pt 38), spans mainly the central region of the gene and its flanking 3 ' sequences. The last one is present in a structurally rearranged karyotype (see subjects in the methods section). The mosaic condition has been confirmed in two patients from which buccal smears were available. Scoring of CREBBP specific signals on both lymphocytes ( $>150$ cells- metaphases and nuclei) and buccal smears (>100 nuclei) gave comparable results in showing an average $20 \%$ of deleted cells in both cases. A fraction of $30 \%$ deleted cells was estimated on the third case for 

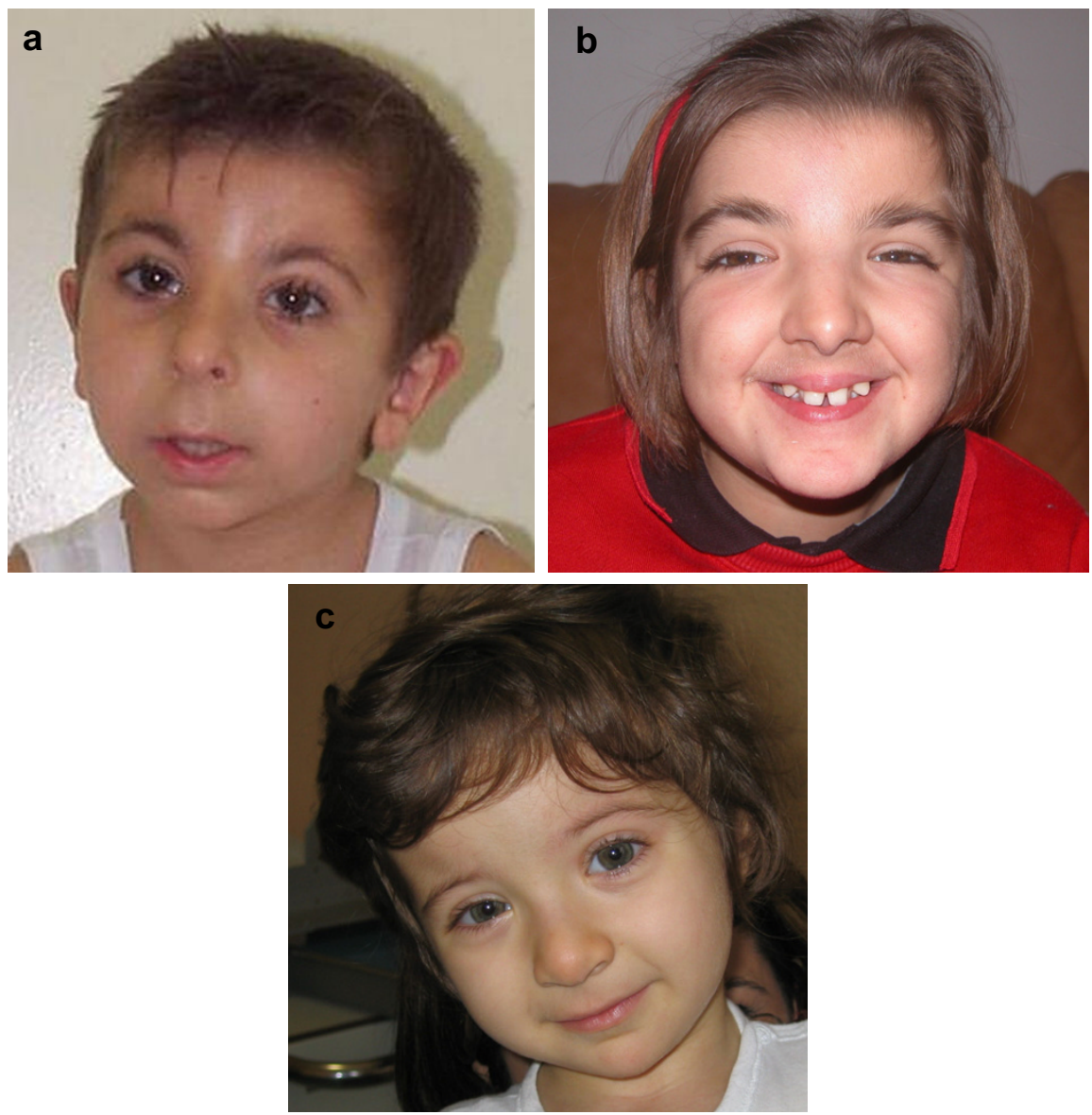

\section{Figure I}

a Patient 16 (negative for CREBBP mutations); b patient 17 (c.37I5_37I6delAA in CREBBP gene); c patient 46 (c.4627G>T in CREBBP gene). The typical RSTS facial features are visible in the three patients.

whom a sample of 300 cells from peripheral blood was scored. Current studies are in progress aimed to evaluating the extent and boundaries of all the deletions. These data will be reported elsewhere. A schematic view of the identified deletions mapped on the encompassed genomic CREBBP region is shown in Figure 3.

Then we screened the remaining 26 patients for point mutations by direct sequencing of 26 PCR genomic fragments encompassing the complete coding sequence and splice sites of CREBBP. We identified 14 sequence alterations of pathogenic significance: all are listed in Table 2 and shown in Figure 4 in their location within exons encoding different CREBBP domains. Although the identified mutations are distributed across the whole gene without apparent hot-spots, six, including two missense (p.Tyr1482Cys; p.Asp1543Tyr), and four truncating mutations (p.Lys1239ValfsX14; p.Gly1479X; p.Asp1543GlnfsX39; p.Pro1655CysfsX89) are clustered within the HAT domain. Out of the remaining mutations, which map outside the HAT domain, five of eight are predicted to lead to premature translation stops upstream of the HAT region, thus indirectly spoiling its function. They comprise p.Arg370X and p.Arg424X in the N-Terminal Transactivation Domain (TAD), p.Gln662X in the CREB Binding Domain and p.Gln1118ProfsX13 and p.Arg1173X in the Bromodomain. The remaining three mutations, p.Arg14Gly in the 5' Nuclear Receptor Binding Domain, p.Ser2015AlafsX25 and p.Gln2022ArgfsX16 in the C-Terminal TAD, do not affect the HAT domain at all. 
Table I: Main clinical signs of the thirty-one Italian RSTS patients.

\begin{tabular}{|c|c|c|c|c|c|c|c|c|c|c|c|}
\hline patient & age & $\operatorname{sex}$ & microcephaly & $\begin{array}{c}\text { facial } \\
\text { features }\end{array}$ & $\mathrm{PMR} / \mathrm{MR}$ & $\begin{array}{l}\text { large/bifid } \\
\text { abducted } \\
\text { thumb }\end{array}$ & $\begin{array}{c}\text { hallux } \\
\text { large/bifid }\end{array}$ & $\begin{array}{l}\text { hallux } \\
\text { valgus/varus }\end{array}$ & $\begin{array}{l}\text { poor } \\
\text { growth }\end{array}$ & malformations & $\begin{array}{l}\text { CREBBP } \\
\text { mutation }\end{array}$ \\
\hline $46 *$ & 3 & $\mathrm{~F}$ & - & + & + moderate & $+/-$ & + & $-/-$ & + & + & missense \\
\hline 42 & 18 & $\mathrm{~F}$ & + & + & + & $+/+$ & + & $-/-$ & + & - & deletion \\
\hline 32 & 4 & $\mathrm{~F}$ & + & + & + & $+/-$ & + & $+/-$ & + & - & nonsense \\
\hline 34 & 2 & $M$ & + & + & + moderate & $+/-$ & - & $-1-$ & + & - & nonsense \\
\hline 15 & II & $M$ & + & + & + severe & $+/-$ & + & $-/-$ & + & + & missense \\
\hline 2 & 10 & $\mathrm{~F}$ & + & + & + moderate & $+/-$ & + & $-/-$ & + & - & insertion \\
\hline 11 & 14 & $M$ & + & + & + & $+/+$ & + & $-/-$ & + & + & nonsense \\
\hline 17 & 14 & $\mathrm{~F}$ & + & + & + & $+/-$ & + & $-/-$ & + & + & deletion \\
\hline 20 & 10 & $\mathrm{~F}$ & + & + & + severe & $+/-$ & + & $-/+$ & + & - & $\begin{array}{l}\text { splice-site } \\
\text { mutation }\end{array}$ \\
\hline 21 & 7 & $M$ & + & + & + & $+/+$ & + & $+/+$ & - & + & deletion \\
\hline 22 & 30 & $\mathrm{~F}$ & + & + & + & $+/+$ & + & $-/-$ & + & - & deletion \\
\hline 23 & 5 & $\mathrm{~F}$ & - & + & + moderate & $+/+$ & + & $-/-$ & - & + & missense \\
\hline 24 & 7 & $\mathrm{~F}$ & - & + & + & $+/-$ & + & $-/-$ & + & - & nonsense \\
\hline 35 & 39 & $M$ & - & + & + & $+/-$ & + & $-/-$ & + & - & nonsense \\
\hline 40 & 26 & $\mathrm{~F}$ & + & nd & + severe & $+/-$ & + & nd & + & - & mosaic del 5' \\
\hline 66 & 6 & $M$ & - & + & + severe & $+/-$ & + & - & - & + & mosaic del 5' \\
\hline 41 & 25 & $\mathrm{~F}$ & + & + & + & $+/+$ & + & $-/-$ & + & - & del mat \\
\hline 38 & 5 & $M$ & - & + & + severe & $-/-$ & + & $-/-$ & - & - & mosaic del 3' \\
\hline 30 & 26 & $\mathrm{~F}$ & + & + & + & $+/+$ & + & $-/-$ & + & - & del mat \\
\hline 36 & 11 & $\mathrm{~F}$ & - & + & + borderline & $+/-$ & - & $-/-$ & - & - & neg \\
\hline 39 & 13 & $M$ & + & + & + & $-/-$ & + & $-/-$ & + & + & neg \\
\hline 43 & II & $M$ & + & + & + servere & nd & nd & nd & - & nd & neg \\
\hline 44 & 21 & $M$ & nd & + & + & $+/+$ & + & $-/-$ & - & - & neg \\
\hline I & 9 & $M$ & + & + & + moderate & $+/-$ & - & $-/-$ & - & - & neg \\
\hline 12 & 17 & $M$ & + & $+/-$ & + moderate & $+/-$ & + & $-/-$ & + & - & neg \\
\hline 14 & 6 & $\mathrm{~F}$ & - & + & + moderate & $+/+$ & + & $-1-$ & - & + & neg \\
\hline 16 & 8 & $M$ & + & + & + moderate & $+/+$ & + & $-/+$ & + & + & neg \\
\hline 25 & 4 & $M$ & - & + & + mild & $+/-$ & + & $-/-$ & - & + & neg \\
\hline 45 & 28 & $\mathrm{~F}$ & nd & + & + moderate & nd & nd & nd & + & nd & neg \\
\hline 28 & 42 & $\mathrm{~F}$ & + & + & + & $+/+$ & + & $-/-$ & - & - & neg \\
\hline 29 & 37 & $M$ & + & + & + & $+/-$ & + & $-/-$ & - & - & neg \\
\hline
\end{tabular}

*development of multiple epiteliomas

Twelve of 14 mutations are novel, while p.Arg370X has been reported by several groups $[8,10,11,17]$ and p.Arg424X has been also recently described [12]. As to the nine different single nucleotide substitutions they consist of five nonsense (pts 24, 35, 11, 32 and 34), three missense (pts 23, 15 and 46) and one splice site mutation (patient 20). Out of the five sequence alterations producing a frameshift in the reading frame, one is a 2 bp-duplication (pt 2) leading to the potential incorporation of 12 anomalous aminoacids, three are deletions sized 1, 2 and 7 nucleotides respectively (pts 22, 17 and 21) predicting 88,13 and 15 anomalous aminoacids and one is a splice site mutation (c.4728+1G>A) affecting IVS 28 donor site. Analysis of this mutation with two different softwares, the Splice Site Prediction by Neural Network (BDGP) [18] and the SpliceView [19] indicated that the splice donor site was suppressed (predicted scores $=0 \%$ ) and a novel candidate donor site 260 bp upstream the mutation was activated. The SpliceView software, also detected a near donor site, 104 bp upstream the splice mutation. We were able to identify CREBBP transcripts from patient 20 also including a smaller transcript, in addition to the normal one (Figure 5). Direct sequencing of the anomalous transcript confirmed the activation of the nearest upstream cryptic donor site predicted by the SpliceView software. The frameshift produced by missplicing should lead to the incorporation of 38 anomalous aminoacids.

All three missense changes, p.Arg14Gly (pt 23), p.Asp1543Tyr (pt 46) and p.Tyr1482Cys (pt 15) were not previously reported. They were established to have occurred de novo and were found absent in 50 (p.Arg14Gly) and in 100 (p.Asp1543Tyr and p.Tyr1482Cys) healthy individuals, respectively. Furthermore all of them affect aminoacids that are highly conserved both through evolution and in the related p300 (Figure 6) [20]. p.Arg14Gly in the 5' Nuclear Receptor Binding Domain is predicted by PSORTII [21] to cause the 
A
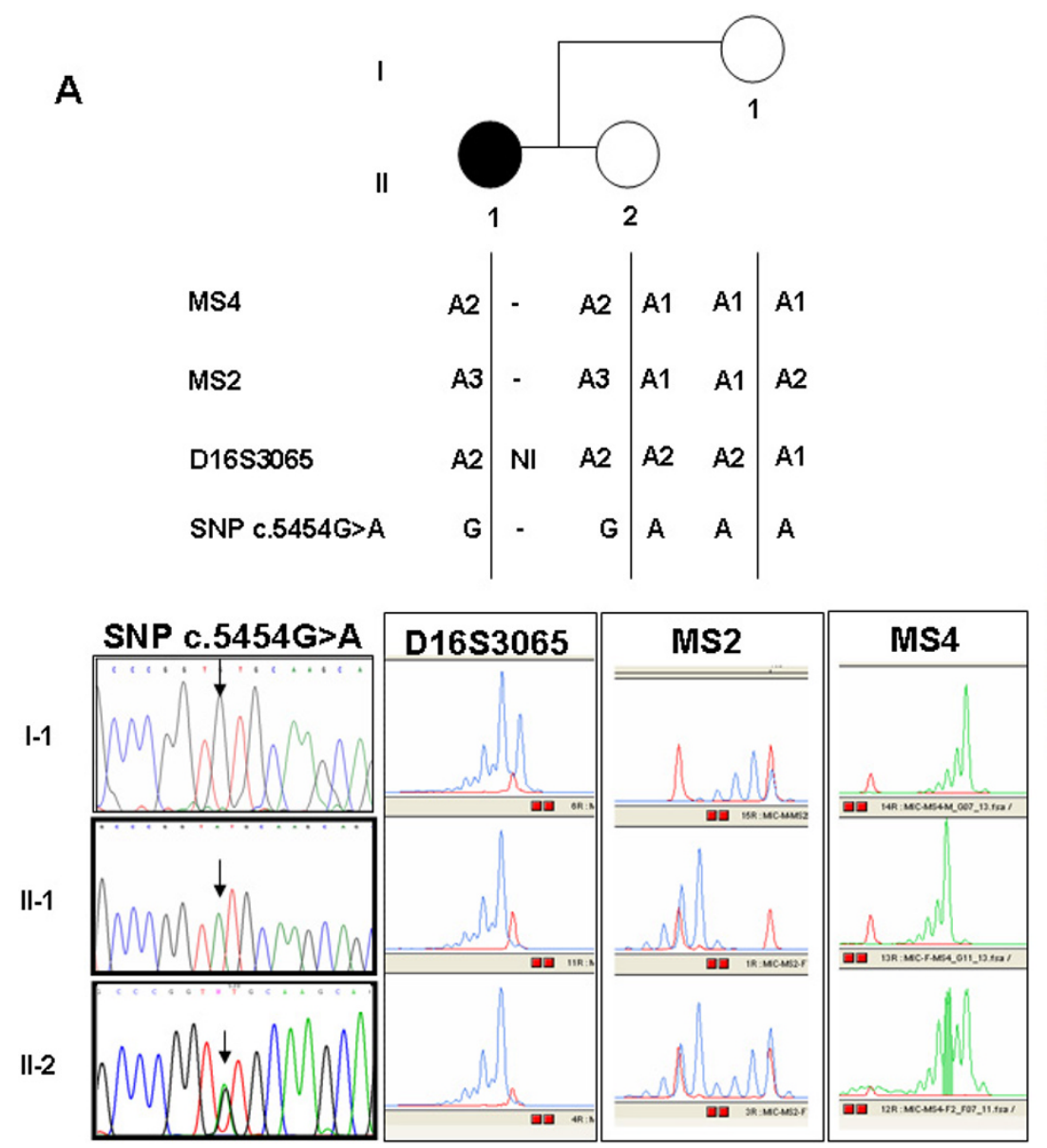

B

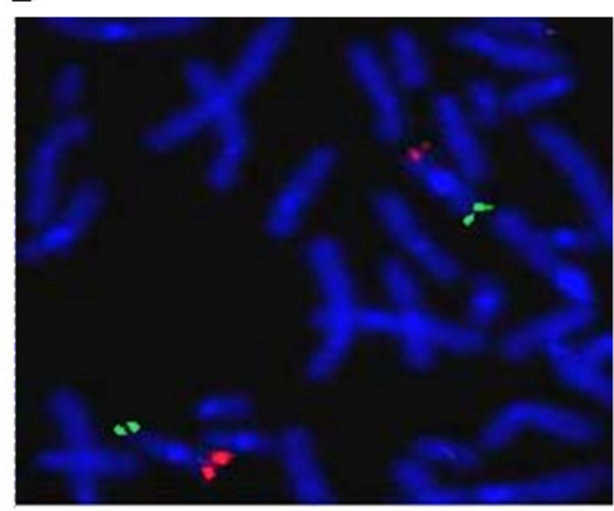

Figure 2

CREBBP deletion in patient 30 detected through: a genotyping of intragenic polymorphic markers (MS4, MS2, DI6S3065 and SNP c.5454G>A) in the available family members allowing haplotype reconstruction; b Double color FISH analysis showing a signal of highly reduced intensity on one chromosome 16p for BAC I072J2, specific for CREBBP (in red). The residual hybridization is accounted for by the size of the genomic clone higher than the deleted region. The control subtelomeric $16 \mathrm{q} B A C$ $566 \mathrm{KI}$ I shows a comparable signal on both chromosomes (in green).

destruction of a monopartite Nuclear Localization Sequence (NLS) (Figure 7). This NLS is a typical monopartite cluster of basic residues, with a conserved pattern starting with Pro, followed by Lys or Arg in 3 out of 5 residues (PKRAKLS) $[22,23]$. In addition the same software predicted another central bipartite Nuclear Localization Signal at 1591 (KKTNKNKSSISRANKKK). Both the NLSs are highly conserved in mouse CREBBP and in p300 protein. p.Tyr1482Cys and the p.Asp1543Tyr are both in the HAT domain. Finally we detected 18 CREBBP polymorphisms, including 12 single nucleotide polymorphisms (SNPs) and a 3'UTR 1 bp-duplication (see Additional file
2). Three of the sequence variations have not been previously reported, including two intronic SNPs (c.3983$22 \mathrm{C}>\mathrm{T}, \mathrm{c} .4560+14 \mathrm{~A}>\mathrm{G}$ ) and the 3 'UTR $7329+25 \mathrm{dupC}$. Conversely the sequence variation $($ c.5933A $>\mathrm{G})$, was reported in other patients with RSTS $[11,12]$. This substitution, causing the p.Asn1978Ser change in a conserved glutamine-rich region, downstream the HAT domain, has been inherited by our patient 22 by his apparently normal mother. The same patient was found to bear a de novo truncating mutation in the exon upstream the p.Asn1978Ser change (c.4963delC). We were able to establish that both mutations occurred on the maternal 


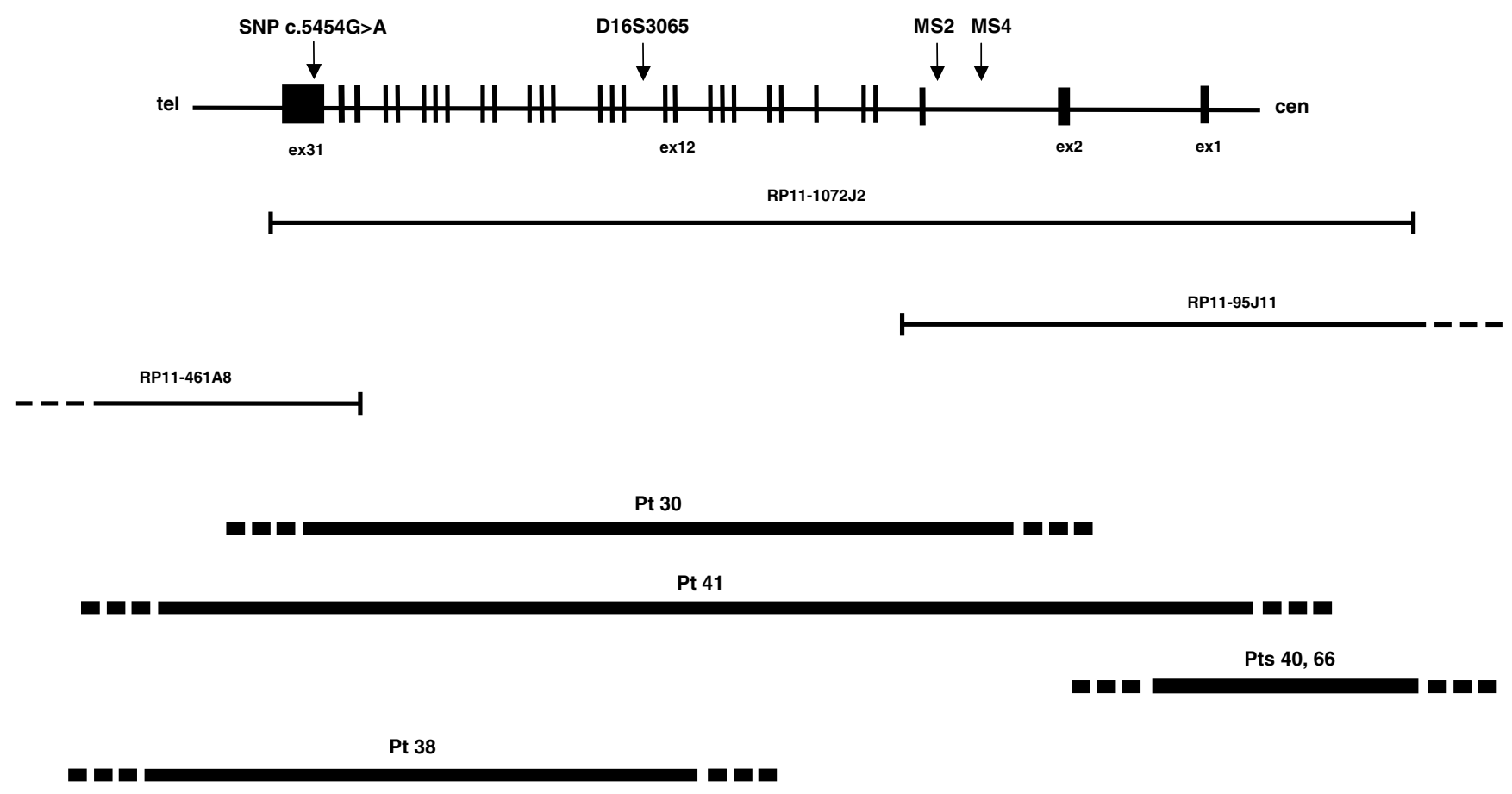

Figure 3

Mapping of the five deletions on the CREBBP genomic region. Top: genomic organization of CREBBP with exons represented by black boxes and introns as connecting lines. The intragenic polymorphic loci are positioned across the gene. Middle: BAC clones used for preliminary FISH analysis, indicated by bars. Bottom: deletions are shown by thick lines; deletion ends containing the breakpoints, are represented by dashed lines.

allele in a cis configuration (data not shown). Moreover we detected the same nucleotide substitution in one of 50 controls analysed.

\section{Discussion}

By investigating 31 RSTS Italian patients using approaches suitable to detect most types of CREBBP mutations we identified bona fide causative lesions in 19 subjects $(61.3 \%)$, a score slightly higher than that recently reported [12]. Complementary application of FISH and microsatellite analysis allowed us to detect five microdeletions, (which refined FISH characterization will be published elsewhere) one of the highest microdeletion rate (16\%) documented so far, a finding that emphasizes possible underestimation of CBP microdeletions in RSTS patients [24-26]. Data achieved using realtime quantitative PCR are consistent with this view [27].

Interestingly this is the first report describing mosaicism for CREBBP deletions, indicating the contribution of mitotic errors to this rare syndrome, that might exploit the same breakpoints of the highly unstable region around intron 2 of $C R E B B P$ evidenced in cancer [28-30]. It should be noted that in one case (pt 38) the CREBBP mosaic dele- tion is in the context of a rearranged karyotype, which may account for the complex phenotype of the patient.

By sequencing all exons and splice sites of the CREBBP gene, we identified on a total of 26 patients 14 different mutations, only two of which have been described $[8,10$ $12,17]$. Neither a predominant type of mutation nor clustering of mutations within the CREBBP gene are apparent. In agreement with previous studies, most mutations (11/ $14,>78 \%$ ) predict premature stop codons $[4,7,8,11,12,17,24]$. Since RSTS is an autosomal dominant trait, it can be assumed that truncating mutations are disease causing mutations making superfluous to determine their de novo origin $[10,11]$. We also detected three putative missense mutations, in which the pathogenic role is much less clear. However, we were able to confirm the de novo origin of all three missense mutations which were found only once among 26 patients analysed and were never observed in a 100 normal controls (50 in the case of patient 23), ruling out that they are unreported CBP polymorphisms. Moreover, when compared with the murine CREBBP (Genbank: S66385) [31], which shows $95 \%$ identity across the whole 2441 aa length of the protein, all the three mutated residues were conserved (Figre 
Table 2: Fourteen causative CREBBP mutations detected by direct sequencing.

\begin{tabular}{|c|c|c|}
\hline PATIENT & MUTATION & TYPE \\
\hline 23 & Ex I: c.40 A>G - p.Arg I4Gly & missense \\
\hline 24 & Ex 4: c.I I08C>T - p.Arg370X (Bartsch et al.2002) & nonsense \\
\hline 35 & Ex 5: c. I 270C>T - p.Arg424X (Bartsch et al.2005) & nonsense \\
\hline 11 & Ex 10: c. 1984C>T - p.Gln662X & nonsense \\
\hline 2 & Ex 17: c.335I_3352dupCC - p.GInIII8ProfsXI3 & insertion \\
\hline 32 & Ex I8: c.35I7C>T - p.Arg|173X & nonsense \\
\hline 17 & Ex 20: c.37I5_37I6delAA - p.Lys | 239ValfsXI4 & deletion \\
\hline 34 & Ex 27: c. $4435 G>T-$ p.Gly|479X & nonsense \\
\hline 15 & Ex 27: c. $4445 \mathrm{~A}>\mathrm{G}-$ p.Tyr I 482Cys & missense \\
\hline 46 & Ex 28: c.4627G>T - p.Asp I543Tyr & missense \\
\hline 20 & Ex 28: c. $4728+\mid \mathrm{G}>\mathrm{A}$ & splice-site mutation \\
\hline 22 & Ex 30: c.4963delC - p.Leul655CysfsX89 & deletion \\
\hline 42 & Ex 3I: c.6043delA - p.Ser20I5AlafsX25 & deletion \\
\hline 21 & Ex 3I: c.6065_607I delAGCAGGC - p.Gln2022AgfsXI6 & deletion \\
\hline
\end{tabular}

In italics: mutations previously described.

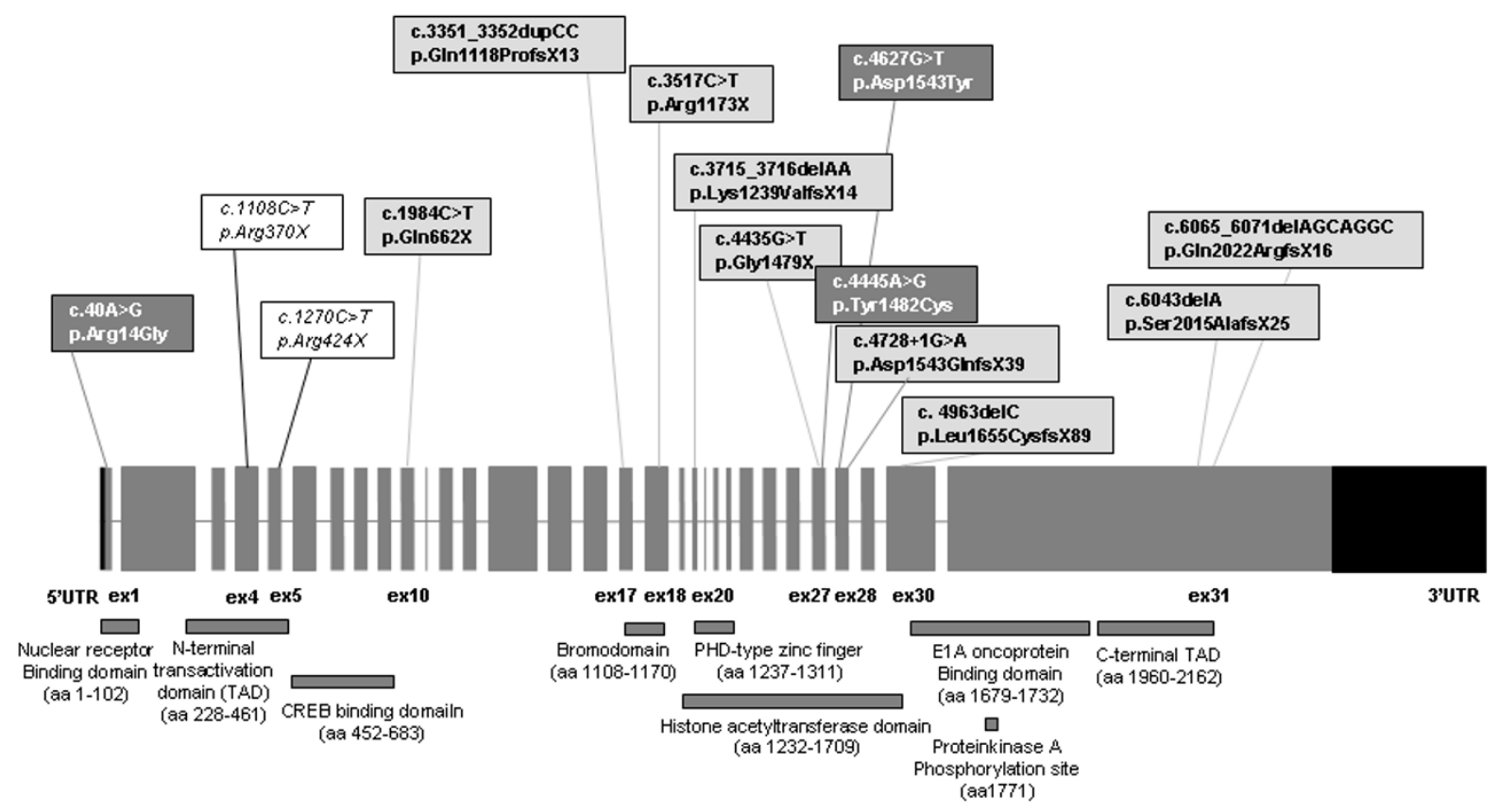

\section{Figure 4}

Location of the 14 causative CREBBP mutations found in this study. Only exons are drawn to scale. The histone acetyl transferase domain and the plant homeodomain (PHD)-type zinc finger are indicated according to Kalkhoven et al. (2003), other domains according to Giles et al. (1997). Truncating mutations are in light-grey, missense mutations in dark-grey and the two previously reported mutations in italics. 
a

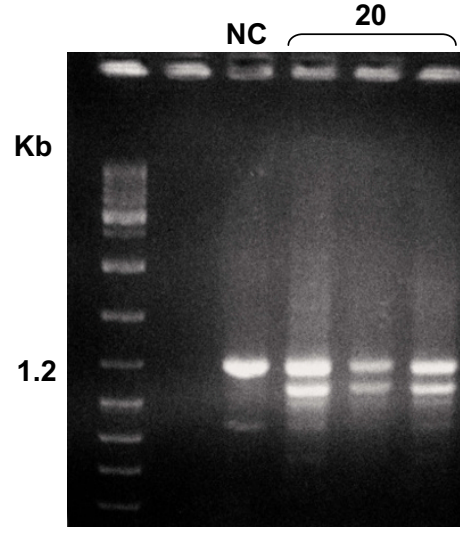

b

\begin{tabular}{lcc} 
& \multicolumn{2}{c}{ DONOR SITE: } \\
& EXON INTRON & SCORE \\
SpliceView & ATG GTGAGG & 88. \\
& AAG GTGATT & 85. \\
& GAG GTACAG & $\mathbf{7 8 .}$ \\
& AAA GTGAGT & 87. \\
Splice Site & ATG GTGAGG & 96 \\
Prediction & GAG GTACAG & $\mathbf{8 6}$ \\
& AAA GTGAGT & 96
\end{tabular}

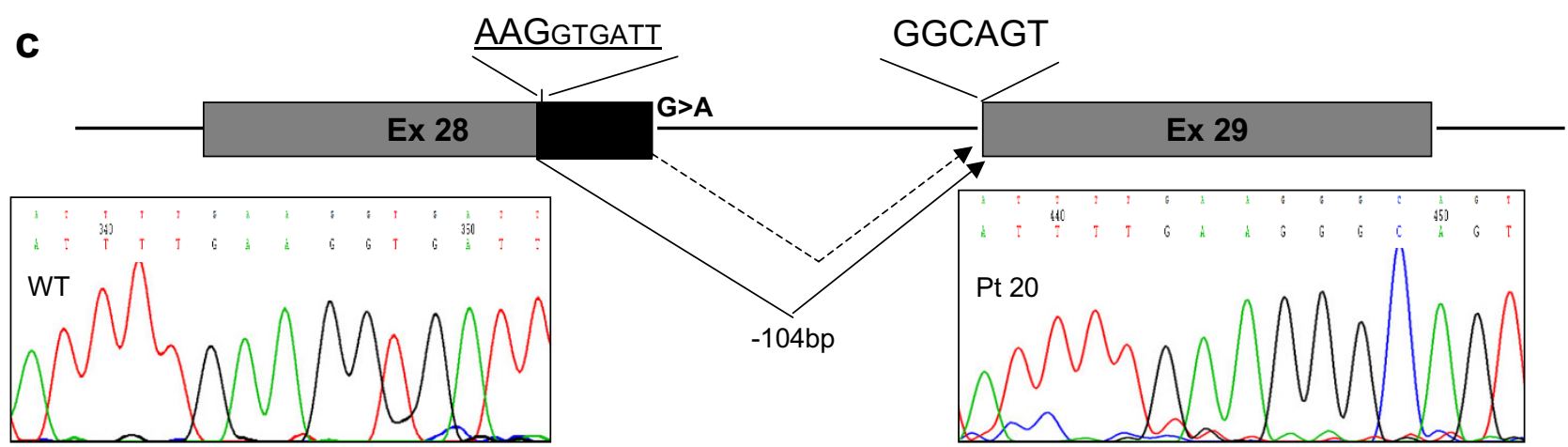

Figure 5

RT-PCR and bioinformatic analysis of the splice site mutation (c.4728+ IG>A) of pt 20. a RT-PCR of the CREBBP fragment including Ex 28 and 29 evidenced an additional smaller fragment (NC: normal control). b bioinformatic analysis with Splice Site Prediction [18] and the SpliceView [19]: the physiological donor site is in bold; the de-novo donor site activated is underscored. c schematic diagram of the misplicing predicted to occur in this patient with the cryptic donor site underscored. On the left electropherogram of the normal donor site; on the right the electropherogram attesting activation of the cryptic donor site.

6). More generally their conservation, both phylogenetic, from fruit fly to C. Elegans, and in the related p300 protein highlights the functional importance of these residues in the protein. Interestingly, two missense mutations are in the HAT domain of CREBBP, where similar mutations were shown to be sufficient to cause the RSTS phenotype as they destroyed the acetyltransferase activity $[7,8]$. Furthermore p.Tyr1482Cys (patient 15) is in the postulated coenzyme A binding site (aminoacids 1459-1541). It may not be fortuitous that the phenotypic presentation of this patient strikingly overlaps the clinical presentation of a second patient (20), carrying a splicing mutation affecting the same domain. Application of RT-PCR allowed to document the deleterious consequence of the splicing mutation and to better interpret the patient's phenotype. Indeed both patients 15 and 20 are affected by severe mental retardation and complete absence of speech. The third missense mutation, p.Arg14Gly, found in patient 23 , is localised in the 5 ' end of the CREBBP protein, where no other missense mutation has been reported. Aminoacids from 1 to 100 are known to be responsible for the
Nuclear-Receptors binding (RAR, GR TXR) [32]. Moreover, according to the PSORTII software the resulting aminoacid change is predicted to disrupt a putative Nuclear Localization Signal. Functional studies of this mutant protein are in progress to determine whether the predicted NLS is necessary for the correct import of the protein into the nucleus. It should be noted that patient 23 has a mild phenotype, characterised by normal growth and absence of microcephaly. This peculiar phenotype might be accounted for by a reduced amount of CREBBP protein in the nucleus because of impaired translocation of the mutated product, rather than by a reduced binding to Nuclear-Receptors.

In this study we detected the c.5933A $>\mathrm{G}$ leading to p.Asn1978Ser change in patient 22, who carries also a de novo truncating mutation (c.4963delC). We were able to establish the cis phase of the two alterations on the maternal allele. The same p.Asn1978Ser change was found in a classic RSTS patient [11] and has been recently described as a recurrent mutation, in a patient displaying an incom- 


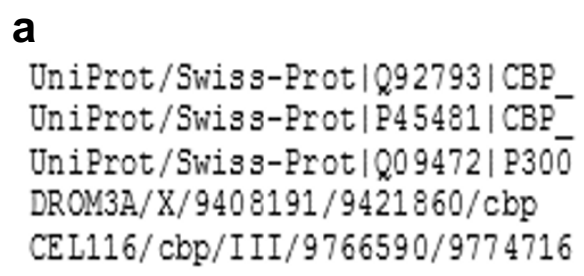

b UniProt/Swis s-Prot|Q92793|CBP_RTAVYHEILIGYLEYVKKLGYVIGHIWACPPSEGDDYIFHCHPPDQKIPK 1495 UniProt/Swis s-Prot | P4 5481| CBP- RIAVYHEILI GYLEYVKKLVYVIAHIWACPPSEGDDYIFHCHPPDQKIPK 1496 UniProt/Swis s-Prot |Q09472 | P300 RTAVYHEILIGYLEYVKKLGYTTGHIWACPPSEGDDYIFHCHPPDQKIPK 1459 DROM3A/X/9408191/9421860/cbp RIAVYHEILLGYMDYVKQLGYTMAHIWACPPSEGDDYIFHCHPT DQKIPK 2116 CEL116/cbp/III/9766590/9774716 RTDVYHELLLGYLDYAKMLGYTMAHIWACPPSEGDDYIFHCHPPEQKIPK 1286

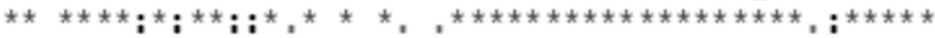

C

UniProt/Swiss-Prot $|092793|$ CBP UniProt/Swiss-Prot |P45481|CBPUniProt/Swiss-Prot|009472|P300 DROM3A/X/9408191/9421860/cbp CEL116/cbp/III/9766590/9774716

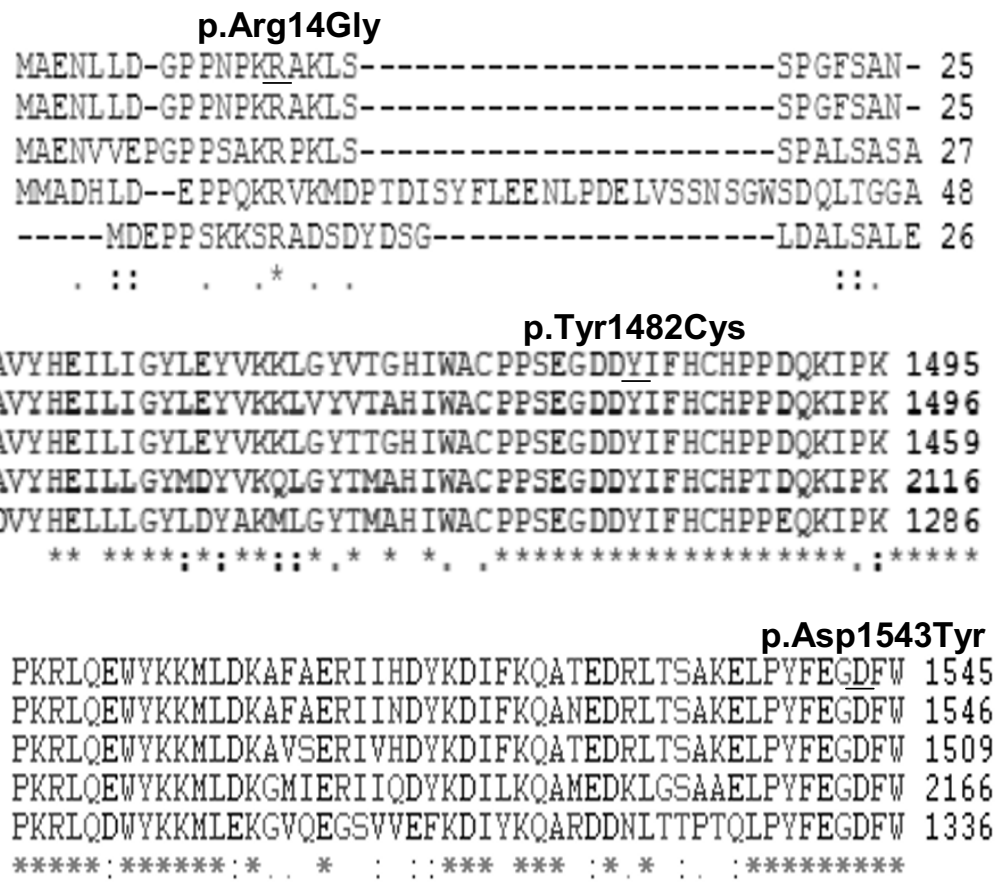

Figure 6

Conservation of amino acids that are predicted by ClustalW [20] to change by three missense mutations identified in this study. a p.Arg I4Gly of pt 23; b p.Tyr 1482Cys of pt I5; c p.Aspl543Tyr of pt 46. The changed residues are conserved in man (GeneBank: Q92793), in mouse (GeneBank: P4548I), in Drosophila melanogaster (DROM3A), in Caenorabditis elegans (CELI I6) and in P300 protein.

plete RSTS phenotype [12]. We detected the same change in our patient 22's healthy mother, and subsequently in one of 50 additional control individuals who were studied: thus we infer it may be counted among CREBBP polymorphisms. Consistent with this view, the resulting amino acid substitution, located outside the HAT domain, affects an aminoacidic residue conserved in the mouse, but not either in fruit fly and C. Elegans, or in p300 (Figure 6). Interestingly, a different change of the same residue (p.Asn1978Asp) has been recently detected in an ovarian and a breast tumour from unrelated individuals. Mutation analysis in germline DNA which could be performed on one of the two individuals showed the same sequence alteration present in the tumours [33]. The authors emphasised that the individual carrying the mutation in the germline does not have the RSTS phenotype, as expected of someone with a CREBBP mutation. All the cumulated evidences indicate the affected CREBBP codon as a highly polymorphic site, resulting in a aminoacidic change which does not disrupt protein function sufficiently to cause RSTS or to predispose to breast and/or ovarian cancer. The overall data might help to interpret this sequence change and to keep it into account in genetic counselling.
Finally, we identified 13 additional sequence variations, which can be added to the list of known CREBBP polymorphisms (see Additional file 2). Interestingly our patients 16 and 25 share five different polymorphisms inherited from their parents, in agreement with a recent report [12]. Also our two families do not appear to be related, but extended genetic histories were not available.

\section{Conclusion}

By combining different techniques we identified a CREBBP mutation in $61.3 \%$ of RSTS cases, a mutation rate higher than that of a recent study [12]. Intragenic microsatellite markers and FISH analysis enabled us to identify one of the highest microdeletion rate $(16 \%)$ documented so far [24-26,34]. No significant correlation could be established between different types of mutation and the clinical presentation and significantly, the patients proved negative to CREBBP mutation screening (both point mutations and deletions) represented the whole RSTS phenotypic spectrum. The overall mutation rate attested by our study and the fact that most CREBBP-negative patients did not display a mild or borderline RSTS clinical presentation provide further indirect evidence on the involvement of genes other than CREBBP in the RSTS phenotype. Indeed, a few CREBBP lesions might have been 


\begin{tabular}{|c|c|}
\hline \multicolumn{2}{|c|}{$\begin{array}{l}\text { pat4: PRKK (4) at } 1080 \\
\text { pat4: RPRK (4) at } 1317 \\
\text { pat4: KPKR (4) at } 1495 \\
\text { pat4: KKKP }(4) \text { at } 1605 \\
\text { pat7: PNPKRAK (3) at } 10 \\
\text { pat7: PKRAKLS (5) at } 12 \\
\text { pat7: PEKRKIII (5) at } 348 \\
\text { pat7: PSQPRKK (3) at } 1077 \\
\text { pat7: PRKKIFK (5) at } 1080 \\
\text { pat7: PKPKRLQ (5) at } 1494\end{array}$} \\
\hline \multicolumn{2}{|c|}{$\begin{array}{l}\text { bipartite:KKTNKNKSSISRANKKK at } 1591 \\
\text { content of basic residues: } 8.8 \% \\
\text { NLS Score: } 3.26\end{array}$} \\
\hline $82.6 \%$ & nuclear \\
\hline $8.7 \%$ & plasma membrane \\
\hline $8.7 \%$ & cytoskeletal \\
\hline
\end{tabular}

\begin{tabular}{|c|c|}
\hline \multicolumn{2}{|c|}{$\begin{array}{l}\text { pat4: PRKK (4) at } 1080 \\
\text { pat4: RPRK (4) at } 1317 \\
\text { pat4: KPKR (4) at } 1495 \\
\text { pat4: KKKP }(4) \text { at } 1605 \\
\text { pat7: PEKRKI }(5) \text { at } 348 \\
\text { pat7: PSQPRKK (3) at } 1077 \\
\text { pat7: PRKKIFK (5) at } 1080 \\
\text { pat7: PKPKRLQ (5) at } 1494 \\
\text { bipartite: KKTNKNKSSISRANKKK at } 1591 \\
\text { content of basic residues: } 8.7 \% \\
\text { NLS Score: } 2.57\end{array}$} \\
\hline $78.3 \%$ & nuclear \\
\hline $8.7 \%$ & plasma membrane \\
\hline $8.7 \%$ & cytoskeletal \\
\hline $4.3 \%$ & cytoplasmic \\
\hline
\end{tabular}

Figure 7

Nuclear Localization signals (NLSs) predicted by PSORTII [2I] analysis. a wild-type CREBBP with three putative NLSs (two monopartite and one bipartite) evidenced by squares: the affected Argl 4 is marked in red; $\mathbf{b}$ Mutated CREBBP of patient 23 with Arg |4Gly substitution with only one predicted NLS. The bottom panels show the cellular localization predictions of wt and mutated proteins.

underestimated, as we ruled out silent exonic alterations affecting splicing enhancers (in the CREBBP-negative patients 14, 16, 25, 28 and 29), but not intronic alterations affecting splicing as analysis at the RNA level was precluded. Similarly the CREBBP promoter could not be tested, as direct sequencing was attempted, but raised technical problems. However the putatively missed $C R E B B P$ alterations likely do not cover the whole fraction of negative cases with a consistent RSTS phenotype. It may be that a few of the screened patients will turn out, upon a closer clinical examination, to have a different syndrome that resembles RSTS. However, considering the larger set of RSTS patients cumulatively ascertained by other groups, yet mutations were not found in more than half their patients as well $[11,12]$. The recent finding of only three mutations of the CREBBP homologue p300 in a series of 91 patients did not prompt us to screen this gene in our much more restricted cohort [10]. However genetic heterogeneity of RSTS patients is documented and lesions in other genes encoding for proteins that interact with
CREBBP in various signal transduction pathways, should be considered.

\section{Competing interests}

The author(s) declare that they have no competing interests.

\section{Authors' contributions}

$\mathrm{AB}$ : main investigator with a direct and coordinating role in experimental work; drafted the manuscript and approved the final version of it.

DM: involved in the collection and critical review of clinical data

CG, PC, FM: performed laboratory investigation concerning both mutation analysis and FISH screening and helped to draft the manuscript

SM e PC: involved in the set up of the mutation screening 
LG, FA, MTD, MLGU, GN, MFB, FF: clinical geneticists providing selected patients with RSTS

AS: supervisor for patients recruitment and clinical diagnosis

LL: involved in design and coordination of the experimental work; drafted the manuscript and approved the final version of it.

All authors read and approved the final manuscript.

\section{Additional material}

\section{Additional file 1}

Additional Table I. Twentysix CREBBP PCR pairs of primer for DNA sequencing of all coding exons of the CREBBP gene, as previously described by Coupry et al. 2002

Click here for file

[http://www.biomedcentral.com/content/supplementary/14712350-7-77-S1.doc]

\section{Additional file 2}

Additional Table II. Thirteen CREBBP polymorphisms detected by direct sequencing. The yet unreported variations are bolded.

Click here for file

[http://www.biomedcentral.com/content/supplementary/14712350-7-77-S2.doc]

\section{Acknowledgements}

We would like to thank the patients and their families for contributing to this study, and our clinical colleagues for referring patients and clinical data, especially Dr Emanuela Lucci-Cordisco (Institute of Medical Genetics, Catholic University, Rome, Italy, pt 40); Dr Alice Pessagno (Department of Infantile Neuropsychiatry, G. Gaslini Children's Research Hospital, Genova, Italy, pt 38); Dr Giovanna Olioso (Divisione di Neuropsichiatria Infantile, Spedali Civili, Brescia, Italy, pt 23). The parents of the children depicted in Figure I have provided consent for the use of the images for publication. This work was supported by a grant from ASM (Associazione Italiana Studio Malformazioni).

\section{References}

I. Rubinstein $\mathrm{JH}$, Taybi $\mathrm{H}$ : Broad thumbs and toes and facial abnormalities. Am J Dis Child 1963, 105:588-608.

2. Miller RW, Rubinstein JH: Tumors in Rubinstein-Taybi Syndrome. Am J Med Genet 1995, 56: I I 2-5.

3. Siraganian PA, Rubinstein JH, Miller RW: Keloids and neoplasms in the Rubinstein-Taybi Syndrome. Med Pediatr Oncol 1989, I 7:485-91.

4. Petrij F, Giles RH, Dauwerse HG, Saris J], Hennekam RC, Masuno M, Tommerup N, van Ommen GJ, Goodman RH, Peters DJ, et al.: Rubinstein-Taybi syndrome is caused by mutations in the transcriptional co-activator CBP. Nature 1995, 376:348-5I.

5. Bannister AJ, Kouzarides T: The CBP coactivator is a histone acetyltransferase. Nature 1996, 384:64|-43.

6. Goodman RH, Smolik S: CBP/p300 in cell growth, transformation, and development. Genes Dev 2000, 14:1553-77.

7. Murata T, Kurokawa R, Krones A, Tatsumi K, Ishii M, Taki T, Masuno M, Ohashi H, Yanagisawa M, Rosenfeld MG, Glass CK, Hayashi Y: Defect of histone acetyltransferase activity of the nuclear transcriptional coactivator CBP in Rubinstein-Taybi syndrome. Hum Mol Genet 200I, I 0: I07I-76.

8. Kalkhoven E, Roelfsema JH, Teunissen $\mathrm{H}$, den Boer A, Ariyurek $\mathrm{Y}$, Zantema A, Breuning MH, Hennekam RC, Peters DJ: Loss of CBP acetyltransferase activity by PHD finger mutations in Rubinstein-Taybi syndrome. Hum Mol Genet 2003, I 2:44I-50.

9. Lundblad JR, Kwok RP, Laurance ME, Harter ML, Goodman RH: Adenoviral EIA-associated protein p300 as a functional homologue of the transcriptional co-activator CBP. Nature 1995, 374:85-88.

10. Roelfsema JH, White SJ, Ariyurek Y, Bartholdi D, Niedrist D, Papadia $F$, Bacino CA, den Dunnen JT, van Ommen G], Breuning MH, Hennekam RC, Peters DJ: Genetic heterogeneity in RubinsteinTaybi syndrome: mutations in both the CBP and EP300 genes can cause disease. Am J Hum Genet 2005, 76:572-80.

II. Coupry I, Roudaut C, Stef M, Delrue MA, Marche M, Burgelin I, Taine L, Cruaud C, Lacombe D, Arveiler B: Molecular analysis of the CBP gene in $\mathbf{6 0}$ patients with Rubinstein-Taybi syndrome. J Med Genet 2002, 39:4I5-2I.

12. Bartsch O, Schmidt S, Richter M, Morlot S, Seemanova E, Wiebe G, Rasi S: DNA sequencing of CREBBP demonstrates mutations in $56 \%$ of patients with Rubistein-Taybi syndrome (RSTS) and in another patients with incomplete RSTS. Hum Genet 2005, I I 7:485-93.

13. Resources for Molecular Cytogenetics [http://www.biolo gia.uniba.it $/ \mathrm{rmc} /]$

14. Lichter P, Cremer T: Chromosome analysis by non-isotopic in situ hybridization. In Human cytogenetics: a practical approach Volume I. 2nd edition. Edited by: Rooney DE, Czepulkowski BH. IRL, Oxford; 1992:157-192.

15. HGMD Home Page [http://www.hgmd.cf.ac.uk/ac/index.php]

16. dbSNP Home Page [http://www.ncbi.nlm.nih.gov/SNP/]

17. Bartsch O, Locher K, Meinecke P, Kress W, Seemanova E, Wagner A, Ostermann K, Rodel G: Molecular studies in 10 cases of Rubinstein-Taybi syndrome, including a mild variant showing a missense mutation in codon 1175 of CREBBP. J Med Genet 2002, 39:4|5-2I.

18. Splice Site Prediction [http://www.fruitfly.org/seq tools/ splice.html]

19. SpliceView pliceview.html]

[http:///25.itba.mi.cnr.it/ webgene/wwws

\section{ClustalW [http://www.ebi.ac.uk/clustalw/]}

21. PSORTII [http://psort.ims.u-tokyo.ac.jp/]

22. Nakai K, Kanehisa M: A knowledge base for predicting protein localization sites in eukaryotic cells. Genomics 1992, |4:897-9|I.

23. Hicks GR, Raikhel NV: Protein import into the nucleus: an integrated view. Annu Rev Cell Dev Biol 1995, I I:I55-188.

24. Bartsch O, Wagner A, Hinkel GK, Krebs P, Stumm M, Schmalenberger B, Bohm S, Balci S, Majewski F: FISH studies in $\mathbf{4 5}$ patients with Rubinstein-Taybi syndrome: deletions associated with polysplenia, hypoplastic left heart and death in infancy. Eur J Hum Genet 1999, 7:748-56.

25. Petrij F, Dauwerse HG, Blough RI, Giles RH, van der Smagt JJ, Wallerstein R, Maaswinkel-Mooy PD, van Karnebeek CD, van Ommen GJ, van Haeringen A, Rubinstein JH, Saal HM, Hennekam RC, Peters DJ, Breuning MH: Diagnostic analysis of the Rubinstein-Taybi syndrome: five cosmids should be used for microdeletion detection and low number of protein truncating mutations. J Med Genet 2000, 37:168-76.

26. Breuning MH, Dauwerse HG, Fugazza G, Saris J], Spruit L, Wijnen H, Tommerup N, van der Hagen CB, Imaizumi K, Kuroki Y, et al.: Rubinstein-Taybi syndrome caused by submicroscopic deletions within 16p 13.3. Am J Hum Genet 1993, 52(2):249-54.

27. Coupry I, Monnet L, Attia AA, Taine L, Lacombe D, Arveiler B: Analysis of CBP (CREBBP) gene deletions in Rubinstein-Taybi syndrome patients using real-time quantitative PCR. Hum Mutat 2004, 23:278-84.

28. Vizmanos JL, Larrayoz MJ, Lahortiga I, Floristan F, Alvarez C, Odero MD, Novo FJ, Calasanz MJ: t(10;16)(q22;p/3) and MORFCREBBP fusion is a recurrent event in acute myeloid leukemia. Genes Chromosomes Cancer 2003, 36:402-5.

29. Schmidt HH, Strehl S, Thaler D, Strunk D, Sill H, Linkesch W, Jager U, Sperr W, Greinix HT, Konig M, Emberger W, Haas OA: RT-PCR and FISH analysis of acute myeloid leukemia with $t(8 ; 16)(p I I ; p \mid 3)$ and chimeric MOZ and CBP transcripts: 
breakpoint cluster region and clinical implications. Leukemia 2004, I 8: I I 5-2I.

30. Rozman M, Camos M, Colomer D, Villamor N, Esteve J, Costa D, Carrio A, Aymerich M, Aguilar JL, Domingo A, Sole F, Gomis F, Florensa L, Montserrat E, Campo E: Type I MOZ/CBP (MYST3/ CREBBP) is the most common chimeric transcript in acute myeloid leukemia with $\mathbf{t}(8 ; 16)(\mathrm{p} \mathrm{I} \mathrm{I;p} \mathrm{I3)} \mathrm{translocation.} \mathrm{Genes}$ Chromosomes Cancer 2004, 40:140-5.

31. Genbank [http://www.ncbi.nlm.nih.gov/Genbank/]

32. Kamei Y, Xu L, Heinzel T, Torchia J, Kurokawa R, Gloss B, Lin SC, Heyman RA, Rose DW, Glass CK, Rosenfeld MG: A CBP integrator complex mediates transcriptional activation and AP-I inhibition by nuclear receptors. Cell 1996, 85:403-I4.

33. Ward R, Johnson M, Shridhar V, van Deursen J, Couch FJ: CBP truncating mutations in ovarian cancer. J Med Genet 1996, 42:5। 4-8.

34. Bartsch O, Rasi S, Delicado A, Dyack S, Neumann LM, Seemanova E, Volleth M, Haaf T, Kalscheuer VM: Evidence for a new contiguous gene syndrome, the chromosome 16p/3.3 deletion syndrome alias severe Rubinstein-Taybi syndrome. Hum Genet in press. 2006, Jun 17

\section{Pre-publication history}

The pre-publication history for this paper can be accessed here:

http://www.biomedcentral.com/1471-2350/7/77/prepub

Publish with Biomed Central and every scientist can read your work free of charge

"BioMed Central will be the most significant development for disseminating the results of biomedical research in our lifetime. "

Sir Paul Nurse, Cancer Research UK

Your research papers will be:

- available free of charge to the entire biomedical community

- peer reviewed and published immediately upon acceptance

- cited in PubMed and archived on PubMed Central

- yours - you keep the copyright

Submit your manuscript here:

http://www.biomedcentral.com/info/publishing_adv.asp 